\title{
Community Pharmacists Attitude Towards Professional Practice in Penang, Malaysia
}

\author{
Abdul Nazer Ali ${ }^{*}$, Sivadasan Shalini ${ }^{2}$, Ping Ng Yen ${ }^{1}$, Prajapati Sunil K${ }^{1}$, Ru Seah Yee ${ }^{1}$, Ying Saw \\ Jia $^{1}$, Thing Tan Ee ${ }^{1}$, Nazer Zulfikar Ahmed ${ }^{3}$ \\ ${ }^{1}$ Faculty of Pharmacy, AIMST University, 08100 Bedong, Kedah Darul Aman, MALAYSIA. \\ ${ }^{2}$ Department of Pharmacy Practice, KMCH College of Pharmacy, Coimbatore, TN, INDIA.
}

${ }^{3}$ Pfizer India Limited, Vishakapatanam, INDIA.

\begin{abstract}
Received: 9 August 2017; Accepted: 29 September 2017

*Correspondence to:

Mr. Abdul Nazer Ali, PhD,

Assoc. Prof., Faculty of Pharmacy, AIMST University, Semeling, 08100, Bedong, Kedah Darul Aman, MALAYSIA.

Email:abdul.nazerali16@gmail.com

\section{Abstract}

Background: Community pharmacists are the health professionals most accessible to the public. Public visit community pharmacies for their daily health care needs. Thus, community pharmacists' attitude towards professional practice is very important. Method: This was a prospective, crosssectional study, using pre-validated questionnaire containing five constructs and 37 items. Each question was given scores 1-5 which denote 1 for very low; 2 for low; 3 for moderate; 4 for high and 5 for very high. The completed questionnaires were analysed using SPSS version 22. Statistical significance for dichotomous data was assessed using chi- square test. Result: Among the 87 participants in managerial activities, 71 (81.6\%) scored good, 14 (16.1\%) scored moderate, 2 (2.3\%) scored poor. In dispensing activities, 71 (81.6\%) scored good, 14 (16.1\%) scored moderate, 2 (2.3\%) scored poor. In pharmaceutical care activities, 56 (64.4\%) scored good, $24(27.6 \%)$ scored moderate, 7 (8.1\%) scored poor. In inter/intra professional activities, $48(55.2 \%)$ scored good, $15(17.2 \%)$ scored moderate and 24 (27.6\%) scored poor. In public health activities, 62 (71.6\%) scored good, 19 (21.8\%) scored moderate and $6(6.9 \%)$ scored poor whereas in maintenance of competency activities, 71 (81.6\%) had scored good, $9(10.3 \%)$ scored moderate and $7(8.1 \%)$ scored poor. Conclusion: Majority of the respondents were having good attitudes towards managerial, dispensing and maintenance of competence activities. However, only minority of the respondents realized the importance of establishing professional relationship with doctors. Continuing education and training programs on professional practice would be one significant approach in equipping community pharmacists with the knowledge, skills and behaviour needed to expand their scope of practice and improving public healthcare outcomes. Key words: Attitude, Community pharmacy, Pharmacist, Professional practice, Malaysia.
\end{abstract}

\section{INTRODUCTION}

Community pharmacists are the health professionals most accessible to the public. Public visit community pharmacies for their daily health care needs. As a community pharmacist, they supply medicines in accordance with a prescription or, when legally permitted, sell them without a prescription. ${ }^{[1]}$ In addition to ensuring an accurate supply of appropriate products, their professional activities also cover counselling of patients at the time of dispensing of prescription and non-prescription drugs, drug information to health professionals, patients and the 
general public, and participation in health-promotion programmes. ${ }^{[2]}$ Thus, pharmacists' attitude towards professional practice is very important. Pharmacists are placed at the first point of contact in the healthcare system due to their easy accessibility. ${ }^{[3]}$ In 1992, the International Pharmaceutical Federation (FIP) presented standards for pharmacy practice under the heading Good pharmacy practice (GPP) in community pharmacies and hospitals' inpatient and outpatient pharmacies. ${ }^{[4]}$ GPP obligates all pharmacists to ensure that the provided services possess the proper quality. ${ }^{[5]}$ GPP guidelines have been prepared by World Health Organization (WHO) and FIP to encourage all countries to develop pharmacy practice activities minimum pharmaceutical care services have an important undisputed positive effect on health care management and costs. ${ }^{[6]}$

\section{METHODS}

A cross-sectional study was conducted on community pharmacies in Penang, Malaysia. A list of 239 registered community pharmacies in Penang area was obtained from the Malaysian Pharmaceutical Society. Data was collected by walk into each randomly selected community pharmacies in Penang area. A pre-validated questionnaire which addressed the standards of practice in managerial activities, dispensing activities, pharmaceutical care activities, inter-intra professional relationships, public health and competence was distributed to the pharmacist of the community pharmacy and the completed questionnaire was collected back, who were asked to score the importance and perceived competence for each of the five domains (sections), on a scale ranging from 1-5 (very low to very high). The questionnaire took about 15 minutes to complete. The inclusion criteria included pharmacists who were legally qualified, registered and work as a community pharmacist, pharmacists who were in attendance in the respective community pharmacies at the time of visit and pharmacists who have given their consent to participate in this study. Intern pharmacists or pharmacists who were on compulsory service programme and pharmacies that had ceased operation or changed locations without forwarding address were excluded.

The questionnaire was adapted from previous studies and slightly modified to suit the objectives of this study. The questionnaire was content validated by six experienced pharmacy lecturers from clinical pharmacy and pharmacy practice unit of a private university and three community pharmacists and modified based on comments. Then the questionnaire was pilot tested, among 12 community pharmacists for internal consistency and stability. Any problems encountered or queries made by the respondents during the pilot study were taken into consideration in formulating the final questionnaire.

All the data obtained were analysed using the Statistical Package for the Social Sciences (SPSS) software, version 22. Descriptive analysis was performed on all the data collected to obtain the frequencies and percentages of occurrence. For numeric data, the means, standard deviations, or medians and IQR were generated. Factors that may be associated with attitude of community pharmacists towards professional practice were determined using Pearson's Chi-square test. P-value of $<.05$ was considered statistically significant.

A total number of 100 questionnaires were distributed and $87(87 \%)$ completed questionnaires were successfully collected back. There were 13 respondents not interested or declined to participate in the study.

\section{Section 1 Managerial and Dispensing activity}

\section{Managerial activity}

All the 87 respondents were distributed with pre-validated questionnaire which included questions under five domains or sections. Section 1, contained 6 questions regarding the managerial and dispensing activities and the responses with frequency and percentage is shown in (Table 3). In this section, minimum possible score was 6 and the maximum score was 30 . The grading of score was done using original Blooms cut-off point, which is $<19$ poor; between 19-23 moderate and $>23$ as good.

\section{Dispensing activity}

The pre-validated questionnaire also included 10 questions regarding dispensing activity with minimum score of 10 and the maximum score of 50 The scoring grade for this section was $<30$ poor; between 31-39 moderate and $>39$ good.

Section 2 Pharmaceutical care activities This section contained eleven questions in pharmaceutical care activities with a minimum scoring mark was 11 and the maximum scoring mark was 55 (Table 3). The grading score for this section was $<33$ poor; between 33-44 moderate and $>44$ good. 
Section 3 Inter/ intra professional activities This section tested three questions in inter/intra professional activities as shown in (Table 3). The minimum scoring grade was 3 and the maximum scoring grade points was 15 . The scoring grade for this section was $<9$ poor; between 9-12 moderate and $>12$ good.

Section 4 Public health activities This section consisted of four questions in public health activities with a minimum score of 4 and the maximum score of 20 . The scoring grade for this section was $<12$ poor; between 12-16 moderate and $>16$ good.

Section 5 Maintenance of competence activities The maintenance of competence activities section consisted of three questions with a minimum score of three and the maximum of 15 . The scoring grade for this section was $<$ 9 poor; between 9-12 moderate and $>12$ good.

\section{ETHICAL CONSIDERATION}

The research proposal along with the interventional tool (Pamphlet), study instruments (questionnaire) and informed consent form (ICF) was submitted to the Institutional Review Board (IRB), AIMST University Human \& Animal Ethical Committee through the faculty of pharmacy ethical committee for study approval before approaching the participants. Signed ICFs were obtained from the participants after explaining the aims and objectives of the study and assured the data provided will be used for research purpose only and high confidence will be maintained at all levels.

\section{RESULTS}

A total number of 87 community pharmacists participated in the study. Among the 87 participants, there were 11 participants in the age group of 21-30 years, 34 participants in the range of 31- 40 years, 27 participants in $41-50$ years, 14 participants in 51- 60 years and 1 participant was more than 60 years of age. The Pearson Chi Square significance observed was found to be $X^{2}(4)=39.61 ; p$ value $<.001$, median age was found to be $40(\mathrm{IQR}=15)$ years. We observed a strong association between ages of the respondents. Among the 87 participants, $45(52 \%)$ were female and $42(48 \%)$ were male participants. The Pearson Chi Square significance observed was found to be $X_{2}$ (1) $=.1 ; p$ value $=.75$. We observed no association between genders of the respondents with their responses to the survey. Among the 87 participants, $9(10 \%)$ participants had
1-5 years of professional experience, 22 (25\%) participants had 6-10 years, 22 (25\%) participants had 11-15 years, 12 (14\%) participants had 16-20 years, 11 (13\%) participants had 21-25 years, 7 (8\%) participants had 26-30 years and 4 $(5 \%)$ participants had more than 30 years of professional experience. The Pearson Chi Square significance observed was found to be $X^{2}(6)=23.95$; $p$ value $<.001$, Median year of experience $=15(\mathrm{IQR}=13)$. We observed a strong association between years of professional experience among the respondents (Table 1).

Section 1 Managerial and dispensing activities (Table 3) shows the frequency and percentage distribution of the participants' response towards the survey domains.

\section{Managerial activities}

Most of the respondents showed an overall $71(82 \%)$ good attitude score, followed by $14(16 \%)$ moderate score and 2 $(2 \%)$ showed poor attitude regarding managerial activities. The Pearson Chi Square significance observed was found to be $X^{2}(2)=83.66 ; p$ value $<.001$. We observed a strong association between attitudes of pharmacists toward managerial activities (Table 2).

\section{Dispensing activity}

Most of the respondents showed an overall $71(82 \%)$ good attitude score, followed by $14(16 \%)$ moderate score and $2(2 \%)$ showed poor attitude score regarding dispensing activities. The Pearson Chi Square significance observed was found to be $X^{2}(2)=93.72 ; p$ value $<.001$. We observed a strong association between attitudes of pharmacist toward dispensing activities (Table 2).

Section 2 Pharmaceutical care activities Most of the respondents showed an overall $56(64 \%)$ good attitude score, followed by $24(28 \%)$ moderate score and $7(8 \%)$ showed poor attitude score regarding pharmaceutical care activities. The Pearson Chi Square significance observed was found to be $X^{2}(2)=42.69 ; p$ value $<.001$. We observed a strong association between attitudes of pharmacist toward pharmaceutical care activities (Table 2).

Section 3 Inter/ intra professional activities Most of the respondents showed an overall 48 (55\%) good attitude score, followed by 15 (17\%) moderate score and 24 (28\%) showed poor attitude score towards inter/ intra professional activities. The Pearson Chi Square significance observed was found to be $X^{2}(2)=20.07 ; p$ value $<.001$. We observed a strong association between attitudes of pharmacist toward inter/ intra professional activities (Table 2). 
Ali et al:: Community Pharmacists Attitude Towards Professional Practice

\begin{tabular}{|c|c|c|c|}
\hline $\begin{array}{l}\text { Variables } \\
\text { Age in Ye }\end{array}$ & No. of Participants & $\%$ & P Value \\
\hline $20-30$ & 11 & 12.6 & \multirow[t]{5}{*}{$<.001^{*}$} \\
\hline $31-40$ & 34 & 39.1 & \\
\hline $41-50$ & 27 & 31.0 & \\
\hline $51-60$ & 14 & 16.1 & \\
\hline$>60$ & 1 & 1.1 & \\
\hline \multicolumn{4}{|l|}{ Gender } \\
\hline Male & 42 & 48.3 & \multirow[t]{2}{*}{.75} \\
\hline Female & 45 & 51.7 & \\
\hline \multicolumn{4}{|c|}{ Professional Experience in Years } \\
\hline $1-5$ & 9 & 10.3 & \multirow[t]{7}{*}{$<.001^{*}$} \\
\hline $6-10$ & 22 & 25.3 & \\
\hline $11-15$ & 22 & 25.3 & \\
\hline $16-20$ & 12 & 13.8 & \\
\hline $21-25$ & 11 & 12.6 & \\
\hline $26-30$ & 7 & 8.0 & \\
\hline$>30$ & 4 & 4.6 & \\
\hline
\end{tabular}

\begin{tabular}{lllllll}
\multicolumn{5}{r}{ Table 2: Overall Attitude Score of the Participants towards Professional Practice. } \\
& Good & Moderate & Poor & $\mathbf{X}^{\wedge} \mathbf{2}$ & df & P Value \\
MA & $71(81.6)$ & $14(16.1)$ & $2(2.3)$ & 83.66 & 2 & $<.001^{*}$ \\
DA & $71(81.6)$ & $14(16.1)$ & $2(2.3)$ & 93.72 & 2 & $<.001^{*}$ \\
PCA & $56(64.4)$ & $24(27.6)$ & $7(8.1)$ & 42.69 & 2 & $<.001^{*}$ \\
IIPA & $48(55.2)$ & $15(17.2)$ & $24(27.6)$ & 20.07 & 2 & $<.001^{*}$ \\
PHA & $62(71.3)$ & $19(21.8)$ & $6(6.9)$ & 59.24 & 2 & $<.001^{*}$ \\
MCA & $71(81.6)$ & $9(10.3)$ & $7(8.1)$ & 91.31 & 2 & $<.001^{*}$
\end{tabular}

Managerial Activities (MA); Dispensing Activities (DA); Pharmaceutical care; Activities (PCA); Inter/Intra Professional Activities (IIPA); Public Health Activities (PHA); Maintenance of Competence Activities (MCA). ${ }^{*}$ Chi square significance at .01 level.

\begin{tabular}{|c|c|c|c|c|c|c|c|}
\hline \multicolumn{2}{|c|}{ Managerial activities } & \multirow{2}{*}{$\begin{array}{l}1(\%) \\
0 \\
(0.0)\end{array}$} & \multirow{2}{*}{$\begin{array}{l}2(\%) \\
0 \\
(0.0)\end{array}$} & \multirow{2}{*}{$\begin{array}{l}3(\%) \\
3 \\
(3.4)\end{array}$} & \multirow{2}{*}{$\begin{array}{l}4(\%) \\
27 \\
(31.0)\end{array}$} & \multirow{2}{*}{$\begin{array}{l}5(\%) \\
57 \\
(65.5)\end{array}$} & \multirow{2}{*}{$\begin{array}{l}\text { Median } \\
5.0\end{array}$} \\
\hline 1. & Ensure pharmacy is well equipped with medicines. & & & & & & \\
\hline 2. & Ensure that medicines are of standard quality. & $\begin{array}{l}0 \\
(0.0)\end{array}$ & $\begin{array}{l}0 \\
(0.0)\end{array}$ & $\begin{array}{l}5 \\
(5.7)\end{array}$ & $\begin{array}{l}20 \\
(23.0)\end{array}$ & $\begin{array}{l}62 \\
(71.3)\end{array}$ & 5.0 \\
\hline 3. & Ensure appropriate storage conditions for medicines. & $\begin{array}{l}0 \\
(0.0)\end{array}$ & $\begin{array}{l}0 \\
(0.0)\end{array}$ & $\begin{array}{l}7 \\
(8.0)\end{array}$ & $\begin{array}{l}26 \\
(29.9)\end{array}$ & $\begin{array}{l}54 \\
(62.1)\end{array}$ & 5.0 \\
\hline 4. & $\begin{array}{l}\text { Ensure that the environment within the pharmacy reflects } \\
\text { a professional setting both in terms of staff and facilities. }\end{array}$ & $\begin{array}{l}2 \\
(2.3)\end{array}$ & $\begin{array}{l}6 \\
(6.9)\end{array}$ & $\begin{array}{l}28 \\
(32.2)\end{array}$ & $\begin{array}{l}28 \\
(32.2)\end{array}$ & $\begin{array}{l}23 \\
(26.4)\end{array}$ & 4.0 \\
\hline 5. & Ensure that the pharmacy has a private area. & $\begin{array}{l}2 \\
(2.3)\end{array}$ & $\begin{array}{l}1 \\
(1.1)\end{array}$ & $\begin{array}{l}19 \\
(21.8)\end{array}$ & $\begin{array}{l}26 \\
(29.9)\end{array}$ & $\begin{array}{l}39 \\
(44.8)\end{array}$ & 5.0 \\
\hline 6. & Ensure that the pharmacy makes a good profit. & $\begin{array}{l}0 \\
(0.0)\end{array}$ & $\begin{array}{l}2 \\
(2.3)\end{array}$ & $\begin{array}{l}13 \\
(14.9)\end{array}$ & $\begin{array}{l}28 \\
(32.2)\end{array}$ & $\begin{array}{l}44 \\
(50.6)\end{array}$ & 5.0 \\
\hline \multicolumn{8}{|c|}{ Dispensing activities } \\
\hline 1. & $\begin{array}{l}\text { Dispense prescription medications only against a } \\
\text { prescription. }\end{array}$ & $\begin{array}{l}3 \\
(3.4)\end{array}$ & $\begin{array}{l}6 \\
(6.9)\end{array}$ & $\begin{array}{l}25 \\
(28.7)\end{array}$ & $\begin{array}{l}15 \\
(17.2)\end{array}$ & $\begin{array}{l}38 \\
(43.7)\end{array}$ & 4.0 \\
\hline
\end{tabular}




\begin{tabular}{|c|c|c|c|c|c|c|c|}
\hline 2. & Assess whether a prescription is legally valid. & $\begin{array}{l}2 \\
(2.3)\end{array}$ & $\begin{array}{l}1 \\
(1.1)\end{array}$ & $\begin{array}{l}21 \\
(24.1)\end{array}$ & $\begin{array}{l}15 \\
(23.0)\end{array}$ & $\begin{array}{l}38 \\
(49.4)\end{array}$ & 4.0 \\
\hline 3. & $\begin{array}{l}\text { Assess the pharmaceutical and pharmacological aspects } \\
\text { of the prescription. }\end{array}$ & $\begin{array}{l}3 \\
(3.4)\end{array}$ & $\begin{array}{l}2 \\
(2.3)\end{array}$ & $\begin{array}{l}5 \\
(5.7)\end{array}$ & $\begin{array}{l}21 \\
(24.1)\end{array}$ & $\begin{array}{l}56 \\
(64.4)\end{array}$ & 5.0 \\
\hline 4. & $\begin{array}{l}\text { Assess the appropriateness of medication for the for } \\
\text { individual prescription. }\end{array}$ & $\begin{array}{l}0 \\
(0.0)\end{array}$ & $\begin{array}{l}2 \\
(2.3)\end{array}$ & $\begin{array}{l}8 \\
(9.2)\end{array}$ & $\begin{array}{l}24 \\
(27.6)\end{array}$ & $\begin{array}{l}53 \\
(60.9)\end{array}$ & 5.0 \\
\hline 5. & $\begin{array}{l}\text { Assess that the medication is most economically available } \\
\text { for the patient. }\end{array}$ & $\begin{array}{l}0 \\
(0.0)\end{array}$ & $\begin{array}{l}0 \\
(0.0)\end{array}$ & $\begin{array}{l}14 \\
(16.1)\end{array}$ & $\begin{array}{l}28 \\
(32.2)\end{array}$ & $\begin{array}{l}45 \\
(51.7)\end{array}$ & 5.0 \\
\hline 6. & Double checking the prescription before dispensing. & $\begin{array}{l}0 \\
(0.0)\end{array}$ & $\begin{array}{l}0 \\
(0.0)\end{array}$ & $\begin{array}{l}2 \\
(2.3)\end{array}$ & $\begin{array}{l}28 \\
(32.2)\end{array}$ & $\begin{array}{l}57 \\
(65.5)\end{array}$ & 5.0 \\
\hline 7. & $\begin{array}{l}\text { Providing the patient with information regarding the } \\
\text { medication. }\end{array}$ & $\begin{array}{l}0 \\
(0.0)\end{array}$ & $\begin{array}{l}0 \\
(0.0)\end{array}$ & $\begin{array}{l}3 \\
(3.4)\end{array}$ & $\begin{array}{l}31 \\
(35.6)\end{array}$ & $\begin{array}{l}53 \\
(60.9)\end{array}$ & 5.0 \\
\hline 8. & $\begin{array}{l}\text { Explain that the patient how to take the medication and for } \\
\text { how long. }\end{array}$ & $\begin{array}{l}0 \\
(0.0)\end{array}$ & $\begin{array}{l}0 \\
(0.0)\end{array}$ & $\begin{array}{l}5 \\
(5.7)\end{array}$ & $\begin{array}{l}19 \\
(21.8)\end{array}$ & $\begin{array}{l}63 \\
(72.4)\end{array}$ & 5.0 \\
\hline 9. & Explain the possible side effects to the patients. & $\begin{array}{l}0 \\
(0.0)\end{array}$ & $\begin{array}{l}2 \\
(2.3)\end{array}$ & $\begin{array}{l}9 \\
(10.3)\end{array}$ & $\begin{array}{l}29 \\
(33.3)\end{array}$ & $\begin{array}{l}47 \\
(54.0)\end{array}$ & 5.0 \\
\hline 10. & $\begin{array}{l}\text { Ensure that the patient has understood the information } \\
\text { provided. }\end{array}$ & $\begin{array}{l}0 \\
(0.0)\end{array}$ & $\begin{array}{l}1 \\
(1.1)\end{array}$ & $\begin{array}{l}9 \\
(10.3)\end{array}$ & $\begin{array}{l}29 \\
(33.3)\end{array}$ & $\begin{array}{l}48 \\
(55.2)\end{array}$ & 5.0 \\
\hline \multicolumn{8}{|c|}{ Pharmaceutical care activities } \\
\hline 1. & $\begin{array}{l}\text { Assess patients' health related problem and medication } \\
\text { requirements. }\end{array}$ & $\begin{array}{l}0 \\
(0.0)\end{array}$ & $\begin{array}{l}1 \\
(1.1)\end{array}$ & $\begin{array}{l}12 \\
(13.8)\end{array}$ & $\begin{array}{l}27 \\
(31.0)\end{array}$ & $\begin{array}{l}47 \\
(54.0)\end{array}$ & 5.0 \\
\hline 2. & $\begin{array}{l}\text { Keep records about the patients' medical condition(s), } \\
\text { medication and progress. }\end{array}$ & $\begin{array}{l}5 \\
(5.7)\end{array}$ & $\begin{array}{l}8 \\
(9.2)\end{array}$ & $\begin{array}{l}25 \\
(28.7)\end{array}$ & $\begin{array}{l}20 \\
(23.0)\end{array}$ & $\begin{array}{l}29 \\
(33.3)\end{array}$ & 4.0 \\
\hline 3. & $\begin{array}{l}\text { Be actively involved in the selection of the most } \\
\text { appropriate medication for the patient. }\end{array}$ & $\begin{array}{l}0 \\
(0.0)\end{array}$ & $\begin{array}{l}3 \\
(3.4)\end{array}$ & $\begin{array}{l}11 \\
(12.6)\end{array}$ & $\begin{array}{l}22 \\
(25.3)\end{array}$ & $\begin{array}{l}51 \\
(58.6)\end{array}$ & 5.0 \\
\hline 4. & $\begin{array}{l}\text { Explain to patients' what he/she should expect from their } \\
\text { medication. }\end{array}$ & $\begin{array}{l}0 \\
(0.0)\end{array}$ & $\begin{array}{l}0 \\
(0.0)\end{array}$ & $\begin{array}{l}12 \\
(13.8)\end{array}$ & $\begin{array}{l}26 \\
(29.9)\end{array}$ & $\begin{array}{l}49 \\
(56.3)\end{array}$ & 5.0 \\
\hline 5. & Monitor patients' progress after dispensing the medicine. & $\begin{array}{l}3 \\
(3.4)\end{array}$ & $\begin{array}{l}7 \\
(8.0)\end{array}$ & $\begin{array}{l}24 \\
(27.6)\end{array}$ & $\begin{array}{l}25 \\
(28.7)\end{array}$ & $\begin{array}{l}28 \\
(32.2)\end{array}$ & 4.0 \\
\hline 6. & $\begin{array}{l}\text { Attempt to identify any drug related problem that patients } \\
\text { may be experiencing. }\end{array}$ & $\begin{array}{l}0 \\
(0.0)\end{array}$ & $\begin{array}{l}2 \\
(2.3)\end{array}$ & $\begin{array}{l}19 \\
(21.8)\end{array}$ & $\begin{array}{l}23 \\
(26.4)\end{array}$ & $\begin{array}{l}43 \\
(49.4)\end{array}$ & 4.0 \\
\hline 7. & $\begin{array}{l}\text { Plan and implement a strategy to resolve any drug related } \\
\text { problems. }\end{array}$ & $\begin{array}{l}0 \\
(0.0)\end{array}$ & $\begin{array}{l}6 \\
(6.9)\end{array}$ & $\begin{array}{l}20 \\
(23.0)\end{array}$ & $\begin{array}{l}25 \\
(28.7)\end{array}$ & $\begin{array}{l}36 \\
(41.4)\end{array}$ & 4.0 \\
\hline 8. & $\begin{array}{l}\text { Have a procedure in place to evaluate the progress and } \\
\text { outcomes of treatment. }\end{array}$ & $\begin{array}{l}6 \\
(6.9)\end{array}$ & $\begin{array}{l}1 \\
(1.1)\end{array}$ & $\begin{array}{l}28 \\
(32.2)\end{array}$ & $\begin{array}{l}22 \\
(25.3)\end{array}$ & $\begin{array}{l}30 \\
(34.5)\end{array}$ & 4.0 \\
\hline 9. & $\begin{array}{l}\text { Communicate the patient's progress on their drug therapy } \\
\text { to their doctor. }\end{array}$ & $\begin{array}{l}5 \\
(5.7)\end{array}$ & $\begin{array}{l}11 \\
(12.6)\end{array}$ & $\begin{array}{l}23 \\
(26.4)\end{array}$ & $\begin{array}{l}19 \\
(21.8)\end{array}$ & $\begin{array}{l}29 \\
(33.3)\end{array}$ & 4.0 \\
\hline 10. & Refer the patient to the doctor when necessary. & $\begin{array}{l}0 \\
(0.0)\end{array}$ & $\begin{array}{l}1 \\
(1.1)\end{array}$ & $\begin{array}{l}6 \\
(6.9)\end{array}$ & $\begin{array}{l}28 \\
(32.2)\end{array}$ & $\begin{array}{l}52 \\
(59.8)\end{array}$ & 5.0 \\
\hline 11. & $\begin{array}{l}\text { Record all professional activities in amanner that allows } \\
\text { access to comprehensive information. }\end{array}$ & $\begin{array}{l}3 \\
(3.4)\end{array}$ & $\begin{array}{l}5 \\
(5.7)\end{array}$ & $\begin{array}{l}16 \\
(18.4)\end{array}$ & $\begin{array}{l}22 \\
(25.3)\end{array}$ & $\begin{array}{l}41 \\
(47.1)\end{array}$ & 4.0 \\
\hline \multicolumn{8}{|c|}{ Inter/intra professional activities } \\
\hline 1. & $\begin{array}{l}\text { Establish a professional relationship with doctors to enable } \\
\text { participation in therapeutic management for patient. }\end{array}$ & $\begin{array}{l}5 \\
(5.7)\end{array}$ & $\begin{array}{l}8 \\
(9.2)\end{array}$ & $\begin{array}{l}29 \\
(33.3)\end{array}$ & $\begin{array}{l}13 \\
(14.9)\end{array}$ & $\begin{array}{l}32 \\
(36.8)\end{array}$ & 4.0 \\
\hline 2. & $\begin{array}{l}\text { Consult with other pharmacists about specific patient } \\
\text { problems. }\end{array}$ & $\begin{array}{l}3 \\
(3.4)\end{array}$ & $\begin{array}{l}4 \\
(4.6)\end{array}$ & $\begin{array}{l}20 \\
(23.0)\end{array}$ & $\begin{array}{l}27 \\
(31.0)\end{array}$ & $\begin{array}{l}33 \\
(37.9)\end{array}$ & 4.0 \\
\hline
\end{tabular}


Ali et al.: Community Pharmacists Attitude Towards Professional Practice

\begin{tabular}{|c|c|c|c|c|c|c|c|}
\hline 3. & $\begin{array}{l}\text { Establish communication with other health care } \\
\text { professional to refer patients with social problems. }\end{array}$ & $\begin{array}{l}2 \\
(2.3)\end{array}$ & $\begin{array}{l}6 \\
(6.9)\end{array}$ & $\begin{array}{l}23 \\
(26.4)\end{array}$ & $\begin{array}{l}23 \\
(26.4)\end{array}$ & $\begin{array}{l}33 \\
(37.9)\end{array}$ & 4.0 \\
\hline \multicolumn{8}{|c|}{ Public health activities } \\
\hline 1. & $\begin{array}{l}\text { Be active in providing general health information to } \\
\text { patients. }\end{array}$ & $\begin{array}{l}0 \\
(0.0)\end{array}$ & $\begin{array}{l}2 \\
(2.3)\end{array}$ & $\begin{array}{l}7 \\
(8.0)\end{array}$ & $\begin{array}{l}29 \\
(33.3)\end{array}$ & $\begin{array}{l}49 \\
(56.3)\end{array}$ & 5.0 \\
\hline 2. & $\begin{array}{l}\text { Provide update, unbiased information on medicines to } \\
\text { patients and doctors. }\end{array}$ & $\begin{array}{l}0 \\
(0.0)\end{array}$ & $\begin{array}{l}1 \\
(1.1)\end{array}$ & $\begin{array}{l}11 \\
(12.6)\end{array}$ & $\begin{array}{l}34 \\
(39.1)\end{array}$ & $\begin{array}{l}41 \\
(47.1)\end{array}$ & 4.0 \\
\hline 3. & Engage in health screening activities. & $\begin{array}{l}2 \\
(2.3)\end{array}$ & $\begin{array}{l}5 \\
(5.7)\end{array}$ & $\begin{array}{l}11 \\
(2.6)\end{array}$ & $\begin{array}{l}29 \\
(33.3)\end{array}$ & $\begin{array}{l}40 \\
(46.0)\end{array}$ & 4.0 \\
\hline 4. & Engage in health promotion activities. & $\begin{array}{l}2 \\
(2.3)\end{array}$ & $\begin{array}{l}4 \\
(4.6)\end{array}$ & $\begin{array}{l}19 \\
(21.8)\end{array}$ & $\begin{array}{l}22 \\
(25.3)\end{array}$ & $\begin{array}{l}40 \\
(46.0)\end{array}$ & 4.0 \\
\hline \multicolumn{8}{|c|}{ Maintenance of competence activities } \\
\hline 1. & Have access to appropriate information services. & $\begin{array}{l}0 \\
(0.0)\end{array}$ & $\begin{array}{l}1 \\
(1.1)\end{array}$ & $\begin{array}{l}12 \\
(13.8)\end{array}$ & $\begin{array}{l}33 \\
(37.9)\end{array}$ & $\begin{array}{l}41 \\
(47.1)\end{array}$ & 4.0 \\
\hline 2. & $\begin{array}{l}\text { Regularly participate in continuous professional } \\
\text { development programmes. }\end{array}$ & $\begin{array}{l}0 \\
(0.0)\end{array}$ & $\begin{array}{l}0 \\
(0.0)\end{array}$ & $\begin{array}{l}14 \\
(16.1)\end{array}$ & $\begin{array}{l}24 \\
(27.6)\end{array}$ & $\begin{array}{l}49 \\
(56.3)\end{array}$ & 5.0 \\
\hline 3. & $\begin{array}{l}\text { Engage in self-assessment of competence and } \\
\text { professional activities. }\end{array}$ & $\begin{array}{l}0 \\
(0.0)\end{array}$ & $\begin{array}{l}0 \\
(0.0)\end{array}$ & $\begin{array}{l}12 \\
(13.8)\end{array}$ & $\begin{array}{l}23 \\
(26.4)\end{array}$ & $\begin{array}{l}52 \\
(59.8)\end{array}$ & 5.0 \\
\hline
\end{tabular}

1=very low; 2=low; 3=moderate; 4=high; 5=very high

Section 4 Public health activities Most of the respondents showed an overall $62(71 \%)$ good attitude score, followed by $19(22 \%)$ moderate score and $6(7 \%)$ showed poor attitude score towards public health activities. The Pearson Chi Square significance observed was found to be $X^{2}(2)$ $=59.24 ; p$ value $<.001$. We observed a strong association between attitudes of pharmacist toward public health activities (Table 2).

Section 5 Maintenance of competence activities Most of the respondents showed an overall $71(82 \%)$ good attitude score, followed by $9(10 \%)$ moderate score and 7 $(8 \%)$ showed poor attitude score towards maintenance of competence activities. The Pearson Chi Square significance observed was found to be $X^{2}(2)=91.31$; value $<.001$. We observed a strong association between attitudes of pharmacist toward maintenance of competence activities (Table 2).

\section{DISCUSSION}

The overall results regarding the attitudes of community pharmacists towards managerial and dispensing activities shows that most of the community pharmacists are playing good role in delivering their professional responsibilities. ${ }^{[7,8]}$ This is not unexpected since these activities are associated with the more traditional functions of pharmacists. ${ }^{[9,}{ }^{10]}$ In fact, the highest scores of the entire section were assigned to the activities of ensuring that the medicines are of good quality. The health outcomes of patients are significantly influenced by the quality of medicines. ${ }^{[1]}$ Thus most of the respondents showed that they are strict towards their responsibility in ensuring that patients get good quality medicines to improve the quality of patients' life. ${ }^{[12]}$ In answering questions relating to dispensing activities, the highest scored was assigned to double checking the prescription before dispensing. ${ }^{[13]}$ Dispensing activities are the key components of the drug-use cycle. This study showed that majority of the respondents was concerned in trying to prevent any medication error during dispensing and aimed for effective outcomes. ${ }^{[14]}$ However, a study conducted in Tasmania, Australia concluded that dispensing errors are occurring well above the regulatory guidelines due to many factors such as high prescription volumes, pharmacist fatigue and overload of work etc. ${ }^{[15]}$

Moreover, from the survey, it is found that respondents also showed good contribution to pharmaceutical care activities. The study in the Republic of Moldova concluded that there was a significantly higher score with regards to the perceived pharmacist's responsibility in engaging in various pharmaceutical care activities. ${ }^{[16]}$ However, the pharmacists approach deeply rooted in the traditional practice of pharmacy pertaining mainly to distributive practice model and is somewhat distant from the other models of practice such as 
pharmaceutical care, drug information and self-care. ${ }^{[17]}$ In answering questions relating to pharmaceutical care, refer the patient to the doctor when necessary scored the highest whereas, keeping records about patients' medical condition(s), medication and progress and communicate patient's progress on their drug therapy to their doctor scored the lowest score. ${ }^{[8]}$ This may reflect that respondents are not fully convinced that pharmaceutical care activities are their responsibility as pharmacist and are still somewhat distant from the concept as a provider of patient care. ${ }^{[18]}$ When looking at the results in the light of the Total Pharmacy Care model for pharmacy practice proposed by Holland and Nimmo, (1999), appears that respondents identify mainly with the distributive practice model and are rather distant from the pharmaceutical care practice model, which includes the tasks of assuming responsibility, on the patient care team, for modifying or dispensing, recommending, monitoring and evaluating a patient's pharmacotherapy, to ensure the outcomes of the pharmacotherapy provided. ${ }^{[19]}$ However, in a recent study conducted in Jordan, they observed the responding pharmacists had a good understanding of pharmaceutical care. ${ }^{[20]}$ They expressed a willingness to implement pharmaceutical care practice but identified a number of barriers for successful implementation. ${ }^{[21-23]}$

When addressing inter/intra professional relationships activities, this study received the lowest scores and seem to be of least concerned by pharmacists among all professional activities. The results reflect the existence of some barriers to establishing such relationships in practice in the community setting. A study conducted revealed that the attitudes of community pharmacists towards the practices in inter/intra professional relationships was the same as that of our study. ${ }^{[24]}$ This is due to the rare interaction between community pharmacists and physicians and dependent on the individuals involved. In most cases, community pharmacists only will communicate with patient's physician if they have any doubt about the prescriptions. ${ }^{[25]}$ Thus, community pharmacists must exercise professional judgement and participate in negotiations to deliver optimum health care to patients. [26] In attitude relating to public health activities, this study showed that most of the respondents were involved actively in providing general health information. Community pharmacists are the health professionals most accessible to the public, hence public always visit community pharmacies for their daily healthcare needs and consult pharmacists if there is any doubt regarding their medication. ${ }^{[27,28]}$ The study conducted in Ghana, revealed that the attitudes of community pharmacists towards the practices in public health activities was almost in line with our study. ${ }^{[29]}$

In the attitude towards maintenance of competence activities, most of the respondents in this study scored well. Most of the respondents engaged in self-assessment of competence and professional activities. They placed higher importance on all professional activities illustrating that they are regularly alert with the current trends in the practice of pharmacy such as professional activities related to the delivery of pharmaceutical care. In the study in Ontario (Canada) revealed that the attitude of community pharmacists towards the practices in maintenance of competence activities was the same as that of this study. ${ }^{[30]}$ This is mainly due to the great advancement of high technology nowadays which enabled the community pharmacists to have an immediate access to database, online information, e-books, etc.

\section{CONCLUSION}

Majority of the respondents were having good attitudes towards managerial, dispensing and maintenance of competence activities. However, only small number of respondents realized the importance of establishing professional relationship with doctors. Continuing education and training programs on professional practice would be one significant approach in equipping community pharmacists with the knowledge, skills and behaviour needed to expand their scope of practice in providing public with better quality of pharmaceutical care and improving public health outcomes.

\section{ACKNOWLEDGMENT}

Authors acknowledge all the community pharmacists who participated in this study by filling up the questionnaires.

\section{CONFLICT OF INTEREST}

None.

\section{FUNDING STATEMENT}

This study was funded by internal grants, 2015 of the AIMST University, Malaysia. 


\section{Ali et al:: Community Pharmacists Attitude Towards Professional Practice}

\section{REFERENCES}

1. Shankar PR. Essential medicines and health products information portal. J Pharmacology Pharmacotherapeutics. 2014;5(1):74.

2. Verma N, Jha KK, Sharma R, Sain-Verma S, Sharma V, Singh R, et al. Role of Pharmacist in New Millenium. Inter J Adv Res Innovation. 2014;2:177-80.

3. Wong FY, Chan FW, You JH, Wong EL, Yeoh EK. Patient self-management and pharmacist-led patient self-management in Hong Kong: A focus group study from different healthcare professionals' perspectives. BMC health Service Res. 2011;11(1):121.

4. The Role of the Pharmacist in the Health Care System: Report of a WHO Consultative Group New Delhi, India 13-16 December 1988; Report of a WHO Meeting Tóquio, Japan, 1993. Geneva, 1994. WHO/PHARM/94.569.

5. World Health Organization: The role of pharmacists in the health care system. (1994) Retrieved from http://apps.who.int/medicinedocs/en/d/ Jh2995e/1.6.2.html accessed on, 2017.

6. Joint FIP/WHO guidelines on good pharmacy practice: standards for quality of pharmacy services. WHO Technical Report Series, No. 961, 2011. Geneva: World Health Organization. 2011.

7. Oparah AC, Eferakeya AE. Attitudes of Nigerian pharmacists towards pharmaceutical care. Pharm World Sci. 2005;27(3):208-14

8. Moullin JC, Sabater-Hernández D, Fernandez-Llimos F, Benrimoj SI. Defining professional pharmacy services in community pharmacy. Res Social Admin Pharm. 2013;9(6):989-95.

9. Schommer JC, Pedersen CA, Doucette WR, Gaither CA, Mott DA. Community pharmacists' work activities in the United States during 2000. J Am Pharm Assoc. 2002;42(3):399-406.

10. Anderson C, Blenkinsopp A, Armstrong M. Feedback from community pharmacy users on the contribution of community pharmacy to improving the public's health: a systematic review of the peer reviewed and non $\square$ peer reviewed literature 1990-2002. Health Expectations. 20041;7(3):191-202.

11. Sackett DL. Evidence-based medicine. In Seminars in perinatology 1997 Feb 28 (Vol. 21:1.pp.3-5). WB Saunders.

12. Smith F. Community pharmacy in Ghana: enhancing the contribution to primary health care. Health Policy and Planning. 2004;19(4):234-41.

13. Peterson GM, Wu MS, Bergin JK. Pharmacists' attitudes towards dispensing errors: their causes and prevention. J Clinical Pharm Therap. 1999;24(1):57-71.

14. Knudsen $P$, Herborg $H$, Mortensen AR, Knudsen M, Hellebek A. Preventing medication errors in community pharmacy: root-cause analysis of transcription errors. Quality Safety Heal Care. 2007;16(4):285-90.

15. Peterson GM, Wu MSH, Bergin JK. Pharmacists' attitudes towards dispensing errors: their causes and prevention. J Clinical Pharm Thera. 1999:24(1):57-71

16. Cordina M, Safta V, Ciobanu A, Sautenkova N. An assessment of community pharmacist's attitudes towards professional practice in the Republic of
Moldova, Pharm Practice. 2008;6(1):1-8.

17. Cooksey JA, Knapp KK, Walton SM, Cultice JM. Challenges to the pharmacist profession from escalating pharmaceutical demand. Health affairs. 2002;21(5):182-8.

18. Pendergast JF, Kimberlin CL, Berardo DH, McKenzie LC. Role orientation and community pharmacists' participation in a project to improve patient care. Social Sci and Med. 1995;40(4):557-65.

19. Holland RW, Nimmo CM. Transitions in pharmacy practice, part1: beyond pharmaceutical care. Am J Health-System Pharm. 1999;56:1758-64.

20. Aburuz S, Al-Ghazawi M, Snyder A. Pharmaceutical care in a communitybased practice setting in Jordan: where are we now with our attitudes and perceived barriers? Inter J Pharm Practice. 2012;20(2):71-9.

21. Mil JW, Boer WO, Tromp TH. European barriers to the implementation of pharmaceutical care. Inter J Pharm Practice. 2001;9(3):163-8.

22. Rossing $\mathrm{C}$, Hansen $\mathrm{EH}$, Krass I. Barriers and facilitators in pharmaceutical care: perceptions and experiences among Danish community pharmacists. J Social Admin Pharm. 2002;19(2):55-64

23. Dunlop JA, Shaw JP. Community pharmacists' perspectives on pharmaceutical care implementation in New Zealand. Pharm World Sci. 2002;24(6):224-30.

24. Bartunek JM. Intergroup relationships and quality improvement in healthcare. BMJ quality and Safety. 2011;20(Suppl 1):i62-6.

25. Wiederholt JB, Clarridge BR, Svarstad BL. Verbal consultation regarding prescription drugs: findings from a state-wide study. Medical Care. 1992:159-73.

26. Edmunds J, Calnan MW. The reprofessionalisation of community pharmacy? An exploration of attitudes to extended roles for community pharmacists amongst pharmacists and General PR actioners in the United Kingdom. Social Sci Med. 2001;53(7):943-55.

27. 27. Cranor CW, Christensen DB. The Asheville Project: short-term outcomes of a community pharmacy diabetes care program. J American Pharm Associ. 2003;43(2):149-59

28. 28. Tinelli $M$, Bond $C$, Blenkinsopp $A$, Jaffray $M$, Watson $M$, Hannaford $P$. Patient evaluation of a community pharmacy medications management service. Ann Pharmacist. 2007;41(12):1962-70.

29. 29. Smith F. Community pharmacy in Ghana: enhancing the contribution to primary health care. Health Policy Plan. 2004;19(4):234-41.

30. 30. Austin Z, Marini A, Desroches B. Use of a learning portfolio for continuous professional development: A study of pharmacists in Ontario (Canada). Pharm Edu. 2005;5

Cite this article as: Ali AN, Shalini S, Yen PN, Sunil KP, Yee RU, Jia YS, Ee TT, Ahmed NZ. Community Pharmacists Attitude Towards Professional Practice in Penang, Malaysia. J Pharm Pract Community Med. 2017;3(4):232-9. 